\title{
Multilevel Floorplanning/Placement for Large-Scale Modules Using $B^{*}$-trees
}

\author{
Hsun-Cheng Lee ${ }^{1}$, Yao-Wen Chang ${ }^{2}$, Jer-Ming $\mathrm{Hsu}^{3}$, and Hannah H. Yang ${ }^{4}$ \\ ${ }^{1}$ Synopsys Inc., Taipei, Taiwan \\ ${ }^{2}$ Department of Electrical Engineering \& Graduate Institute of Electronics Engineering, National Taiwan University, Taipei 106, Taiwan \\ ${ }^{3}$ National Center for High-Performance Computing, Hsinchu, Taiwan \\ ${ }^{4}$ Strategic CAD Labs, Intel Corporation, Hillsboro, OR 97124, USA
}

\begin{abstract}
We present in this paper a multilevel floorplanning/placement framework based on the $\mathrm{B}^{*}$-tree representation, called $M B^{*}$-tree, to handle the floorplanning and packing for large-scale building modules. The MB*-tree adopts a two-stage technique, clustering followed by declustering. The clustering stage iteratively groups a set of modules based on a cost metric guided by area utilization and module connectivity, and at the same time establishes the geometric relations for the newly clustered modules by constructing a corresponding $\mathrm{B}^{*}$-tree for them. The declustering stage iteratively ungroups a set of the previously clustered modules (i.e., perform tree expansion) and then refines the floorplanning/placement solution by using a simulated annealing scheme. In particular, the MB*-tree preserves the geometric relations among modules during declustering, which makes the $\mathrm{MB}^{*}$-tree an ideal data structure for the multilevel floorplanning/placement framework. Experimental results show that the $\mathrm{MB}^{*}$-tree obtains significantly better silicon area and wirelength than previous works. Further, unlike previous works, $\mathrm{MB}^{*}$-tree scales very well as the circuit size increases.
\end{abstract}

\section{Categories and Subject Descriptors}

B. 7.2 [Integrated Circuits]: Design Aids-Placement and Routing; J.6 [Computer Applications]: Computer-Aided Engineering-Computer-Aided Design

\section{General Terms}

Algorithms, Design, Performance, Theory

\section{Keywords}

Floorplanning, Multilevel Framework, Lagrangian Relaxation

\section{Introduction}

Design complexities are growing at a breathtaking speed with the continued improvement of the nanometer IC technologies. On one hand, designs with hundreds of million transistors are already in production, IP modules are widely reused, and a large number of buffer blocks are used for delay optimization as well as noise reduction in very deep-submicron interconnect-driven floorplanning $[1,7,11,13,21]$, which all drive the need of a tool to handle large-scale building modules. On the other hand, the highly competitive IC market requires faster design convergence, faster incremental design turnaround, and better silicon area utilization. Efficient and effective design methodology and tools capable of placing and optimizing large-scale modules are essential for such large designs.

Many floorplan representations have been proposed [5, 9, 14, 15, 16, 18, 19 , $20,22]$ in the literature. However, traditional floorplanning/placement algorithms do not scale well as the design size, complexity, and constraints increase, mainly due to their inflexibility in handling non-slicing floorplans, and/or intrinsically non-hierarchical data structures (representations). The B*-tree, in contrast, has been shown an efficient, effective, and flexible data structure for non-slicing floorplans [5]. It is particularly suitable for representing a non-slicing floorplan with large-scale modules and for creating or incrementally updating a floorplan. What is more important, its binary-tree based structure directly corresponds to the framework of a hierarchical, divide-and-conquer scheme, and thus the properties inherited from the structure can substantially facilitate the operations for multilevel large-scale building module floorplanning/placement.

Based on the $\mathrm{B}^{*}$-tree representation, we present in this paper a multilevel floorplanning/placement framework, called $M B^{*}$-tree, to handle the floorplanning and packing for large-scale building modules with high efficiency and quality. MB*tree is inspired by the success of the multilevel framework in graph/circuit partitioning such as Chaco [10], hMetis [12], and ML [2], placement such as MPL [4],

${ }^{*}$ This work was partially supported by the National Science Council of Taiwan by Grant No. NSC-91-2215-E-002-038. E-mails: hclee@synopsys.com; ywchang@cc.ee.ntu.edu.tw; gis89530@cis.nctu.edu.tw; hyang@ichips.intel.com.

Permission to make digital or hard copies of all or part of this work for personal or classroom use is granted without fee provided that copies are not made or distributed for profit or commercial advantage and that copies bear this notice and the full citation on the first page. To copy otherwise, to republish, to post on servers or to redistribute to lists, requires prior specific permission and/or a fee.

DAC 2003, June 2-6, 2003, Anaheim, California, USA

Copyright 2003 ACM 1-58113-688-9/03/0006 ...\$5.00 and routing such as MRS [6], MR [17], and MARS [8]. Unlike multilevel partitioners and placers, however, multilevel floorplanning poses unique difficulties as the shapes of modules to be clustered together can significantly affect the area utilization of a floorplan, and a floorplan design within a cluster needs to be explored along with the global floorplan optimization. The clustering approach also helps to directly address floorplan congestion and timing issues, since different clustering algorithms can be developed to localize inter-module communication and reduce critical path length.

The MB*-tree algorithm adopts a two-stage technique, clustering followed by declustering. The clustering stage iteratively groups a set of modules (could be basic modules and/or previously clustered modules) based on a cost metric guided by area utilization and module connectivity, and at the same time establishes the geometric relations for the newly clustered modules by constructing a corresponding $\mathrm{B}^{*}$-tree. The clustering procedure repeats until a single cluster containing all modules is formed, denoted by a one-node $\mathrm{B}^{*}$-tree that bookkeeps the entire multilevel clustering information. For soft modules, we apply Lagrangian relaxation during clustering to determine the module shapes. Then, the declustering stage iteratively ungroups a set of the previously clustered modules (i.e., expanding a node into a subtree according to the $\mathrm{B}^{*}$-tree topology constructed at the clustering stage), and then apply simulated annealing to refine the floorplanning/placement solution based on a cost metric defined by area utilization and wirelength. The refinement shall lead to a "better" B*-tree structure that guides the declustering at the next level. It is important to note that we always keep only one $\mathrm{B}^{*}$-tree for processing at each iteration, and the $\mathrm{MB}^{*}$-tree preserves the geometric relations among modules during declustering (i.e., the tree expansion), which makes the $\mathrm{MB}^{*}$-tree an ideal data structure for the multilevel floorplanning/placement framework.

Experimental results show that the $\mathrm{MB}^{*}$-tree scales very well as the circuit size increases while the famous previous works, sequence pair, O-tree, and $\mathrm{B}^{*}$-tree alone, do not. For circuit sizes ranging from 49 to 9,800 modules and from 408 to 81,600 nets, the $\mathrm{MB}^{*}$-tree consistently obtains high-quality floorplans with dead spaces of less than $3.7 \%$ in empirically linear runtime, while sequence pair, O-tree, and $\mathrm{B}^{*}$-tree can handle only up to 196,196 , and 1,960 modules in the same amount of runtime and result in the dead spaces of as large as 13.00\% (@ 196 modules), 9.86\% (@196 modules), and 27.33\% (@1960 modules), respectively. We also performed experiments based on a large industrial design with 189 modules and 9777 nets. The results show that our MB*-tree algorithm obtained significantly better silicon area and wirelength than previous works.

The remainder of this paper is organized as follows. Section 2 formulates the module floorplanning/placement problem. Section 3 gives a brief overview on the B*-tree representation. Section 4 presents our two-stage algorithm, clustering followed by declustering. Section 5 presents our approach for handling soft modules. Section 6 gives the experimental results.

\section{Problem Formulation}

Let $M=\left\{m_{1}, m_{2}, \ldots, m_{n}\right\}$ be a set of $n$ rectangular modules. Each module $m_{i} \in M$ is associated with a three tuple $\left(h_{i}, w_{i}, a_{i}\right)$, where $h_{i}, w_{i}$, and $a_{i}$ denote the width, height, and aspect ratio of $m_{i}$, respectively. The area $A_{i}$ of $m_{i}$ is given by $h_{i} w_{i}$, and the aspect ratio $a_{i}$ of $m_{i}$ is given by $h_{i} / w_{i}$, Let $r_{i, \min }$ and $r_{i, \max }$ be the minimum and maximum aspect ratios, i.e., $h_{i} / w_{i} \in\left[r_{i, \min }, r_{i, \max }\right]$. A placement (floorplan) $P=\left\{\left(x_{i}, y_{i}\right) \mid m_{i} \in M\right\}$ is an assignment of rectangular modules $m_{i}$ 's with the coordinates of their bottom-left corners being assigned to $\left(x_{i}, y_{i}\right)$ 's so that no two modules overlap (and $h_{i} / w_{i} \in\left[r_{i, \min }, r_{i, \max }\right], \forall i$ ). We consider in this paper both hard and soft modules. A hard module is not flexible in its shape but free to rotate. A soft module is free to rotate and change its shape within the range $\left[r_{i, \min }, r_{i, \max }\right]$. The objective of placement/floorplanning is to minimize a specified cost metric such as a combination of the area $A_{t o t}$ and wirelength $W_{t o t}$ induced by the assignment of $m_{i}$ 's, where $A_{\text {tot }}$ is measured by the final enclosing rectangle of $P$ and $W_{t o t}$ the summation of half the bounding box of pins for each net.

\section{Review of the $B *$-tree Representation}

Given a compacted placement $P$ that can neither move down nor move left (called an admissible placement [9]), we can represent it by a unique $\mathrm{B}^{*}$-tree $T$ [5]. (See Figure 1(b) for the $\mathrm{B}^{*}$-tree representing the placement of Figure 1(a).) A B*tree is an ordered binary tree (a restriction of O-tree with faster and more flexible operations) whose root corresponds to the module on the bottom-left corner. Using 
the depth-first search (DFS) procedure, the $\mathrm{B}^{*}$-tree $T$ for an admissible placement $P$ can be constructed in a recursive fashion. Starting from the root, we first recursively construct the left subtree and then the right subtree. Let $R_{i}$ denote the set of modules located on the right-hand side and adjacent to $m_{i}$. The left child of the node $n_{i}$ corresponds to the lowest module in $R_{i}$ that is unvisited. The right child of $n_{i}$ represents the lowest module located above $m_{i}$, with its $x$-coordinate equal to that of $m_{i}$

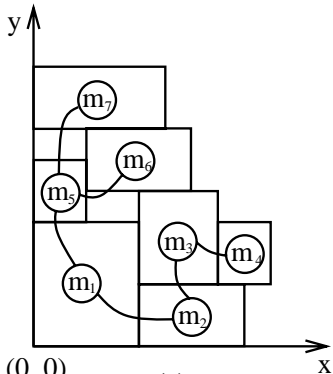

(a)

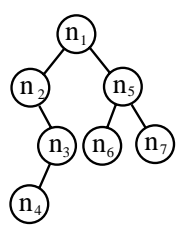

(b)
Figure 1: An admissible placement and its corresponding $\mathrm{B}^{*}$-tree.

The $\mathrm{B}^{*}$-tree keeps the geometric relationship between two modules as follows If node $n_{j}$ is the left child of node $n_{i}$, module $m_{j}$ must be located on the righthand side of $m_{i}$, with $x_{j}=x_{i}+w_{i}$. Besides, if node $n_{j}$ is the right child of $n_{i}$, module $m_{j}$ must be located above module $m_{i}$, with the $x$-coordinate of $m_{j}$ equal to that of $m_{i}$; i.e., $x_{j}=x_{i}$. Also, since the root of $T$ represents the bottom-left module, the coordinate of the module is $\left(x_{\text {root }}, y_{\text {root }}\right)=(0,0)$.

Inheriting from the nice properties of ordered binary trees, the $\mathrm{B}^{*}$-tree is simple, efficient, effective, and flexible for handling non-slicing floorplans. It is particularly suitable for representing a non-slicing floorplan with various types of modules and for creating or incrementally updating a floorplan. What is more important, its binary-tree based structure directly corresponds to the framework of a hierarchical scheme, which makes it a superior data structure for multilevel large-scale building module floorplanning/placement.

\section{The MB*-tree Algorithm}

In this section, we shall present our $\mathrm{MB}^{*}$-tree algorithm for multilevel largescale building module floorplanning/placement. As mentioned earlier, the algorithm adopts a two-stage approach, clustering followed by declustering, by using the $\mathrm{B}^{*}$-tree representation.

The clustering operation results in two types of modules, namely primitive modules and cluster modules. A primitive module $m$ is a module given as an input (i.e., $m \in M$ ) while a cluster one is created by grouping two or more primitive modules. Each cluster module is created by a clustering scheme $\left\{m_{i}, m_{j}\right\}$, where $m_{i}\left(m_{j}\right)$ denotes a primitive or a cluster module.

In the following subsections, we detail the clustering and declustering algorithms for hard modules.

\subsection{Clustering}

In this stage, we iteratively group a set of (primitive or cluster) modules until a single cluster is formed (or until the number of cluster modules is smaller than a threshold) based on a cost metric of area and connectivity. We shall first consider the clustering metric.

The clustering metric is defined by the two criteria: area utilization (dead space) and the connectivity density among modules.

- Dead space: The area utilization for clustering two modules $m_{i}$ and $m_{j}$ can be measured by the resulting dead space $s_{i j}$, representing the unused area after clustering $m_{i}$ and $m_{j}$. Let $s_{t o t}$ denote the dead space in the final floorplan $P$. We have $s_{t o t}=A_{t o t}-\sum_{m_{i} \in M} A_{i}$, where $A_{i}$ denotes the area of module $m_{i}$ and $A_{\text {tot }}$ the area of the final enclosing rectangle of $P$. Since $\sum_{m_{i} \in M} A_{i}$ is a constant, minimizing $A_{t o t}$ is equivalent to minimizing the dead space stot.

- Connectivity density: Let the connectivity $\epsilon_{j j}$ denote the number of nets between two modules $m_{i}$ and $m_{j}$. The connectivity denisty $d_{i j}$ between two (primitive or cluster) modules $m_{i}$ and $m_{j}$ is given by

$$
d_{i j}=c_{i j} /\left(n_{i}+n_{j}\right),
$$

where $n_{i}\left(n_{j}\right)$ denotes the number of primitive modules in $m_{i}\left(m_{j}\right)$. Often a bigger cluster implies a larger number of connections. The connectivity density considers not only the connectivity but also the sizes of clusters between two modules to avoid possible biases.

Obviously, the cost function of dead space is for area optimization while that of connectivity density is for timing and wiring area optimization. Therefore, the metric for clustering two (primitive or cluster) modules $m_{i}$ and $m_{j}$, $\phi:\left\{m_{i}, m_{j}\right\} \rightarrow \Re^{+} \cup\{0\}$, is then given by

$$
\phi\left(\left\{m_{i}, m_{j}\right\}\right)=\alpha \hat{s}_{i j}+\frac{\beta K}{\hat{d}_{i j}},
$$

where $\hat{s}_{i j}$ and $K / \hat{d}_{i j}$ are respective normalized costs for $s_{i j}$ and $K / d_{i j}, \alpha, \beta$ and $K$ are user-specified parameters/constants.

Based on $\phi$, we cluster a set of modules into one at each iteration by applying the aforementioned methods until a single cluster containing all primitive modules is formed or the number of modules is smaller than a given threshold (and thus can be easily handled by the classical program). During clustering, we shall record how two modules $m_{i}$ and $m_{j}$ are clustered into a new cluster module $m_{k}$. If $m_{i}$ is placed left to (below) $m_{j}$, then $m_{i}$ is horizontally (vertically) related to $m_{j}$, denoted by $m_{i} \rightarrow(\uparrow) m_{j}$. If $m_{i} \rightarrow(\uparrow) m_{j}$, then $n_{j}$ is the left (right) child of $n_{i}$ in its corresponding $\mathrm{B}^{*}$-tree. The relation for each pair of modules in a cluster is established and recorded in the corresponding $\mathrm{B}^{*}$-subtree during clustering. It will be used for determining how to expand a node into a corresponding $\mathrm{B} *$-subtree during declustering.

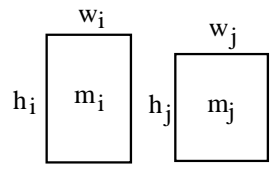

(a)

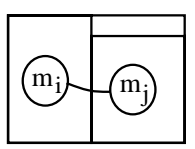

(b)

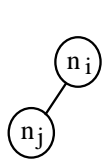

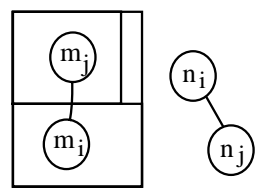

(c)
Figure 2: The relation of two modules and their clustering. (a) Two candidate modules $m_{i}$ and $m_{j}$. (b) The clustering and the corresponding B*-subtree for the case where $m_{i}$ is horizontally related to $m_{j}$. (c) The clustering and the corresponding $\mathrm{B}^{*}$-subtree for the case where $m_{i}$ is vertically related to $m_{j}$.

\subsection{Declustering}

We shall first introduce the metric used in simulated annealing for refining floorplan/placement solutions. The declustering metric is defined by the two creiteria: area utilization (dead space) and the wirelength among modules.

- Dead space: Same as that defined in Section 4.1.

- Wire length: The wirelength of a net is measured by half the bounding box of all the pins of the net, or by the length of the center-to-center interconnections between the modules if no pin positions are specified. The wirelength for clustering two modules $m_{i}$ and $m_{j}, w_{i j}$, is measured by the total wirelength interconnecting the two modules. The total wirelength in the final floorplan $P, w_{t o t}$, is the summation of the length of the wires interconnecting all modules.

Obviously, the cost function of dead space is for area optimization while that of wirelength is for timing and wiring area optimization. Therefore, the metric for refining a floorplan solution during declustering, $\psi_{i j}:\left\{m_{i}, m_{j}\right\} \rightarrow \Re^{+} \cup\{0\}$, is then given by

$$
\psi_{i j}=\gamma \hat{s}_{i j}+\delta \hat{w}_{i j},
$$

where $\hat{s}_{i j}$ and $\hat{w}_{i j}$ are respective normalized costs for $s_{i j}$ and $w_{i j}$, and $\gamma$ and $\delta$ are user-specified parameters.

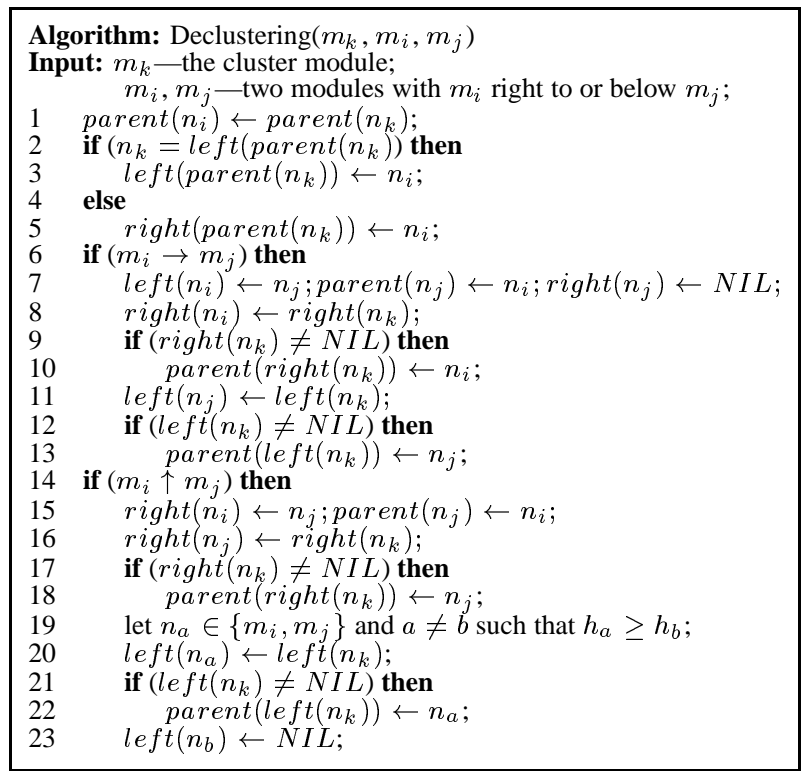

Figure 3: The declustering algorithm. 


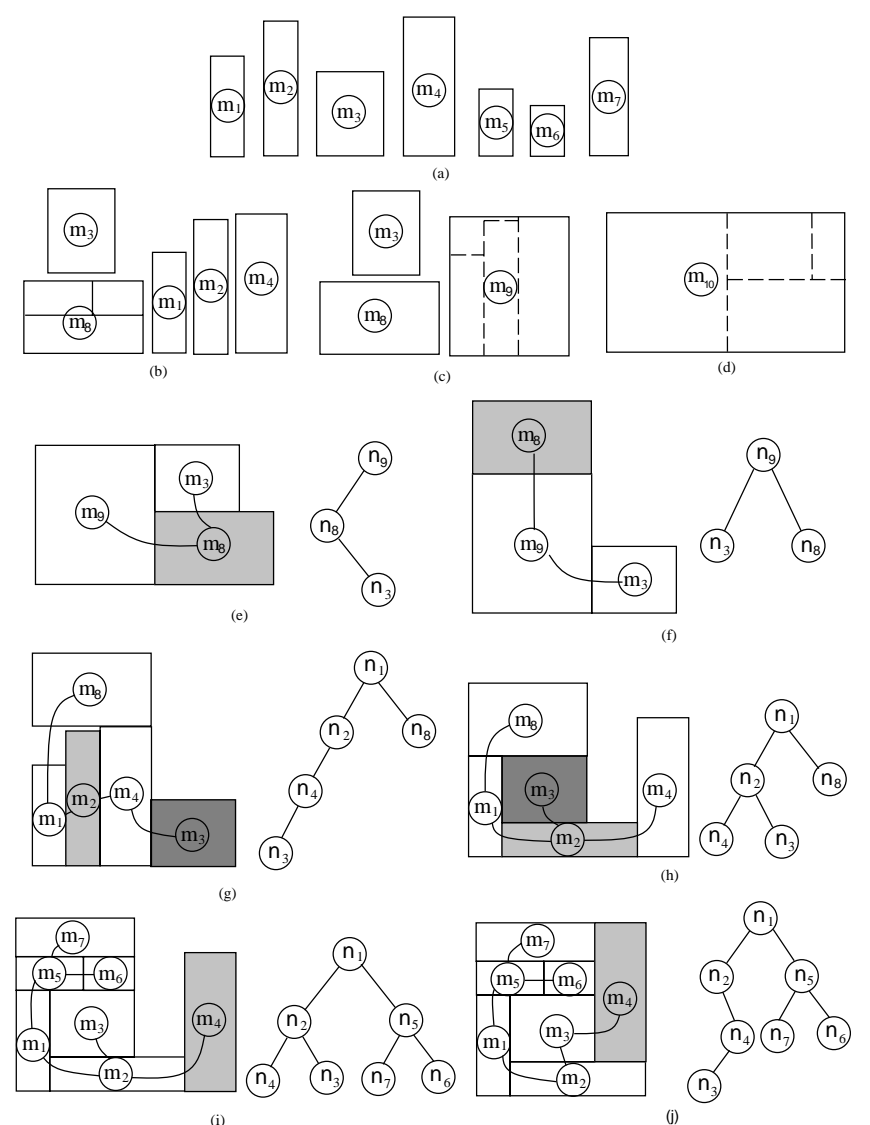

Figure 4: (a) Given seven modules, $m_{i}$ 's, $1 \leq i \leq 7$. (b) Cluster $m_{5}, m_{6}$, and $m_{7}$ into $m_{8}$. (c) Cluster $m_{1}, m_{2}$, and $m_{4}$ into $m_{9}$. (d) Cluster $m_{3}, m_{8}$, and $m_{9}$ into $m_{10}$. (e) Decluster $m_{10}$ to $m_{3}, m_{8}$, and $m_{9}$. (f) Perform Op2 for $m_{8}$. (g) Decluster $m_{9}$ to $m_{1}, m_{2}$, and $m_{4}$. (h) Perform Op1 and Op2 for $m_{2}$ and $m_{3}$, respectively. (i) Decluster $m_{8}$ to $m_{5}, m_{6}$, and $m_{7}$. (j) Perform Op2 for $m_{4}$.

The declustering stage iteratively ungroups a set of previously clustered modules (i.e., expand a node into a subtree according to the $\mathrm{B}^{*}$-tree constructed at the clustering stage) and then refines the floorplan solution based on simulated annealing.

Figure 3 shows the algorithm for declustering a cluster module $m_{k}$ into two modules $m_{i}$ and $m_{j}$ that are clustered into $m_{k}$ at the clustering stage. Without loss of generality, we make $m_{i}$ right to or below $m_{j}$. In Algorithm Declustering (see Figure 3), parent $\left(n_{i}\right)$, right $\left(n_{i}\right)$, and $\operatorname{left}\left(n_{i}\right)$ denote the parent, the right child, and the left child of node $n_{i}$ in a $\mathrm{B}^{*}$-tree, respectively. Line 1 updates the parent of $n_{k}$ as that of $n_{i}$. Lines 2-5 make $n_{i}$ a left (right) child if $n_{k}$ is a left (right) child. Lines 6-13 deal with the case where $m_{i}$ is horizontally related to $m_{j}$. If $m_{i} \rightarrow m_{j}$, then $n_{j}$ is the left child of $n_{i}$ and thus we update the corresponding links in Line 7. Lines 8-10 (11-13) update the links associated the right (left) child of $n_{k}$. Similarly, lines 14-23 cope with the case where $m_{i}$ is vertically related to $m_{j}$.

Theorem 1 Each declustering operation takes $O(1)$ time, and the overall declustering stage takes $O(|M|)$ time, where $|M|$ is the number of input primitive modules.

\subsection{Simulated Annealing}

We proposed a simulated annealing based algorithm to refine the solution at each level of declustering. We apply the following three operations to perturb a multilevel B*-tree (a feasible solution) to another.

- Op1: Rotate a module.

- Op2: Move a module to another place.

- Op3: Swap two modules.

Op1 exchanges the width and height of a module. Op2 deletes a node of a B*-tree and inserts it into another position. Op 3 deletes two nodes and inserts them into the corresponding positions in the $\mathrm{B}^{*}$-tree. Obviously, $\mathrm{Op} 2$ and $\mathrm{Op} 3$ need to perform the deletion and insertion operations on a $\mathrm{B}^{*}$-tree, which takes $O(h)$ time, where $h$ is the height of the $\mathrm{B}^{*}$-tree.

The simulated annealing algorithm starts by a $\mathrm{B}$-tree produced during declustering. Then it perturbs a $\mathrm{B}^{*}$-tree (a feasible solution) to another $\mathrm{B}^{*}$-tree by Op1, Op2, and/or Op3 until a predefined "frozen" state is reached. At last, we transform the resulting $\mathrm{B}^{*}$-tree to the corresponding final admissible placement.

\subsection{The Overall MB*-tree Algorithm}

The MB*-tree algorithm integrates the aforementioned three algorithms. We first perform clustering to reduce the problem size level by level and then enter the declustering stage. In the declustering stage, we perform floorplanning for the modules at each level using the simulated annealing based algorithm $\mathrm{B}^{*}$-tree SA.

Figure 4 illustrates an execution of the $\mathrm{MB}^{*}$-tree algorithm. For explanation, we cluster three modules each time in Figure 4. Figure 4(a) lists seven modules to be packed, $m_{i}$ 's, $1<i<7$. Figures 4(b)-(d) illustrate the execution of the clustering algorithm. Figures 4(b) shows the resulting configuration after clustering $m_{5}, m_{6}$, and $m_{7}$ into a new cluster module $m_{8}$ (i.e., the clustering scheme of $m_{8}$ is $\left.\left\{\left\{m_{5}, m_{6}\right\}, m_{7}\right\}\right)$. Similarly, we cluster $m_{1}, m_{2}$, and $m_{4}$ into $m_{9}$ by using the clustering scheme $\left\{\left\{m_{2}, m_{4}\right\}, m_{1}\right\}$. Finally, we cluster $m_{3}, m_{8}$, and $m_{9}$ into $m_{10}$ by using the clustering scheme $\left\{\left\{m_{3}, m_{8}\right\}, m_{9}\right\}$. The clustering stage is thus done, and the declustering stage begins, in which simulated annealing is applied to do the floorplanning. In Figure 4(e), we first decluster $m_{10}$ into $m_{3}, m_{8}$, and $m_{9}$ (i.e., expand the node $n_{10}$ into the $\mathrm{B}^{*}$-subtree illustrated in Figure 4(e)). We then move $m_{8}$ to the top of $m_{9}$ (perform Op2 for $m_{8}$ ) during simulated annealing (see Figure 4(f)). As shown in Figure 4(g), we further decluster $m g$ into $m_{1}, m_{2}$, and $m_{4}$, and then rotate $m_{2}$ and move $m_{3}$ on top of $m_{2}$ (perform Op1 on $m_{2}$ and Op2 on $m_{3}$ ), resulting in the configuration shown in Figure 4(h). Finally, we decluster $m_{8}$ shown in Figure 4(i) to $m_{5}, m_{6}$, and $m_{7}$, and move $m_{4}$ to the right of $m_{3}$ (perform Op2 for $m_{4}$ ), which results in the optimum placement shown in Figure 4(j).

\section{Handling Soft Modules}

In this section, we present our approach for handling soft modules. We first apply Lagrangian relaxation [23] to cluster soft modules at the clustering stage while keeping declustering the same as before. We then propose a network-flow based algorithm for projecting Lagrange multipliers to satisfy their optimality conditions.

\subsection{Formulation}

Let $M=\left\{m_{1}, m_{2}, \ldots, m_{n}\right\}$ be a set of $n$ primitive soft modules. Each primitive soft module $m_{i} \in M$ is associated with a three tuple $\left(h_{i}, w_{i}, a_{i}\right)$, where $h_{i}, w_{i}$, and $a_{i}$ denote the width, height, and aspect ratio of $m_{i}$, respectively. The area $A_{i}$ of $m_{i}$ is given by $h_{i} w_{i}$, and the aspect ratio $a_{i}$ of $m_{i}$ is given by $h_{i} / w_{i} \in$ $\left[r_{i, \min }, r_{i, \max }\right]$. Let $L_{i}=\sqrt{A_{i} / r_{i, \min }}$ and $U_{i}=\sqrt{A_{i} / r_{i, \max }}$ denote the minimum and the maximum width of $m_{i}$, respectively. We have $h_{i}=A_{i} / w_{i}$ and $L_{i} \leq w_{i} \leq U_{i}$.

A cluster module $m_{c}$ is composed of a set of primitive soft modules $M_{p} . m_{c}$ can be reshaped via reshaping the modules in $M_{p}$ without violating the relations of the modules in $M_{p}$. We create two dummy modules $m_{s}$ and $m_{t}$ and set $x_{s}=0$, $y_{s}=0, w_{s}=0$, and $h_{s}=0$. Then we construct a horizontal and a vertical constraint subgraphs of $m_{c}$, denoted by $G_{h c}$ and $G_{v c}$, respectively. $G_{h c}$ and $G_{v c}$ are constructed as follows:

- For $m_{s}$ and $m_{t}$, create two vertices $v_{s}$ and $v_{t}$ in both $G_{h c}$ and $G_{v c}$.

- For each $m_{p} \in M_{p}$, create a vertex $v_{p}$ in $G_{h c}$ and $G_{v c}$.

- For each $m_{p}, m_{q} \in M_{p}$, if $m_{p}$ is left to (below) $m_{q}$, create an edge $e(p, q)$ from $v_{p}$ to $v_{q}$ in $G_{h c}\left(G_{v c}\right)$.

- For each $m_{p}$ which is placed at the left boundary (bottom boundary), create an edge $e\left(v_{s}, v_{p}\right)$ from $v_{s}$ to $v_{p}$ in $G_{h c}\left(G_{v c}\right)$.

- For each $m_{p}$ which is placed at the right boundary (top boundary), create an edge $e\left(v_{p}, v_{t}\right)$ from $v_{p}$ to $v_{t}$ in $G_{h c}\left(G_{v c}\right)$.

If $x_{p}+w_{p} \leq x_{q}, \forall e(p, q) \in G_{h c}$ and $y_{p}+\frac{A_{p}}{w_{p}} \leq y_{q}, \forall e(p, q) \in G_{v c}$ are satisfied, the relations of the modules in $M_{p}$ will not be violated. Figure 5 illustrates how to construct $G_{h i}$ and $G_{v i}$ and what corresponding constraints must be satisfied. Figure 5(a) gives a cluster modules $m_{c}$ with the cluster scheme $\left\{\left\{m_{1}\right.\right.$, $\left.\left.m_{2}\right\}, m_{3}\right\}$. Figure 5(b) shows the corresponding constraint subgraphs $G_{h c}$ and $G_{v c}$ of $m_{c}$. Figure 5(c) shows the constraints to ensure that no relation of modules is violated. Thus, it impies that $w_{c} \geq x_{t}$, and $h_{c} \geq y_{t}$.

At level $i$, Let $M^{i}=\left\{m_{1}^{i}, m_{2}^{i}, \ldots, m_{n_{i}}^{i}\right\}$ denote the set of cluster modules. For each $m_{j}^{i} \in M^{i},\left(x_{j}^{i}, y_{j}^{i}\right)$ denote the coordinate of its bottom-left corner, and $h_{j}^{i}$ and $w_{j}^{i}$ denote the height and width of $m_{j}^{i}$, respectively. For convenience, we additionally create two variables, $x_{n_{i}+1}^{i}$ and $y_{n_{i}+1}^{i}$, which denote the estimated height and width of the chip at level $i$, respectively. Thus, the estimated area of the chip at level $i$ equals $x_{n_{i}+1}^{i} y_{n_{i}+1}^{i}$. To estimate wirelength, we adopt the quadratic of the length of the complete graph of pins in a net, and take the center of a module as the location of a pin, if the pins are not assigned during floorplanning. Let $E^{i}$ denote the set of nets at level $i$. For a net $e_{j}^{i} \in E^{i}, e_{j}^{i}$ can be represented as a set of the modules $\left\{m_{k}^{i} \mid e_{j}^{i}\right.$ has a pin connecting to $\left.m_{k}^{i}\right\}$. Thus, the estimated wirelengh $\varpi_{j}^{i}$ of a net $e_{j}^{i} \in E^{i}$ is defined by

$$
\begin{aligned}
\varpi_{j}^{i}= & \sum_{m_{p}^{i}, m_{q}^{i} \in e_{j}^{i}}\left(\left(\left(x_{p}^{i}+w_{p}^{i} / 2\right)-\left(x_{q}^{i}+w_{q}^{i} / 2\right)\right)^{2}\right. \\
& \left.+\left(\left(y_{p}^{i}+h_{p}^{i} / 2\right)-\left(y_{q}^{i}+h_{q}^{i} / 2\right)\right)^{2}\right) .
\end{aligned}
$$




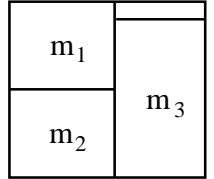

$\mathrm{m}_{\mathrm{c}}$

(a)

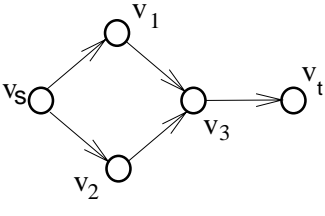

$\mathrm{G}_{\mathrm{hc}}$

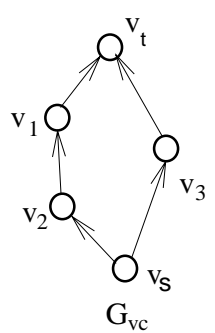

(b)

$$
\begin{gathered}
0 \leq x_{1} \\
0 \leq x_{2} \\
x_{1}+w_{1} \leq x_{3} \\
x_{2}+w_{2} \leq x_{3} \\
x_{3}+w_{3} \leq x_{t} \\
0 \leq y_{2} \\
0 \leq y_{3} \\
y_{2}+\frac{A_{2}}{w_{2}} \leq y_{1} \\
y_{1}+\frac{A_{1}}{w_{1}} \leq y_{t} \\
y_{1}+\frac{A_{1}}{w_{1}} \leq y_{t}
\end{gathered}
$$

Figure 5: (a) A cluster modules $m_{c}$ with the cluster scheme $\left\{\left\{m_{1}, m_{2}\right\}, m_{3}\right\}$. (b) $m_{c}$ 's corresponding constraint subgraphs $G_{h c}$ and $G_{v c}$. (c) The constraints to ensure that no relation of modules is violated.

We use the cost function $\phi^{\prime}$ to guide the clustering of soft modules:

$$
\phi^{\prime}(x, y)=\alpha x_{n_{i}+1}^{i} y_{n_{i}+1}^{i}+\beta \sum_{e_{j}^{i} \in E^{i}} \varpi_{j}^{i},
$$

where $\alpha$ and $\beta$ are nonnegative user-defined parameters, and $\varpi_{j}^{i}$ denotes the estimated wirelength of a net $e_{j}^{i}$. In the formulation of clustering for soft modules, we have the constraints that all modules are not overlapped and must be laid in the chip (i.e. $x_{j}^{i}+w_{j}^{i} \leq x_{n_{i}+1}^{i}$ and $y_{j}^{i}+h_{j}^{i} \leq y_{n_{i}+1}^{i}$ ). Therefore, we can formulate the problem of clustering for soft modules, called $C S$, as follows:

$$
\begin{gathered}
\quad \alpha x_{n_{i}+1}^{i} y_{n_{i}+1}^{i}+\beta \sum_{e_{j}^{i} \in E^{i}} \varpi_{j}^{i} \\
\text { Minimize } \\
x_{j}^{i}+w_{j}^{i} \leq x_{n_{i}+1}^{i}, y_{j}^{i}+h_{j}^{i} \leq y_{n_{i}+1}^{i}, \forall 1 \leq j \leq n_{i}, \\
x_{p}+w_{p} \leq x_{q}, \forall e(p, q) \in G_{h j} \forall 1 \leq j \leq n_{i}, \\
x_{t_{j}} \leq w_{j}^{i}, y_{t_{j}} \leq h_{j}^{i}, \forall 1 \leq j \leq n_{i} \\
y_{p}+\frac{A_{p}}{w_{p}} \leq y_{q}, \forall e(p, q) \in G_{v j} \forall 1 \leq j \leq n_{i}, \\
L_{i} \leq w_{i} \leq U_{i}, \forall 1 \leq i \leq n,
\end{gathered}
$$

\section{where $\alpha$ and $\beta$ are nonnegative user-defined parameters.}

\subsection{Lagrangian Relaxation}

Then, the Lagrangian relaxation subproblem associated with the multiplier $\overrightarrow{\mathcal{P}}=$ $(\vec{\kappa}, \vec{\eta}, \vec{\lambda}, \vec{\mu}, \vec{r}, \vec{s})$, denoted by $L R S /(\overrightarrow{\mathcal{P}})$, can be defined as follows:

$$
\begin{aligned}
\text { Minimize } \quad & \alpha x_{n_{i}+1}^{i} y_{n_{i}+1}^{i}+\beta \sum_{e_{j}^{i} \in E^{i}} \varpi_{j}^{i} \\
& +\sum_{j=1}^{n_{i}} \kappa_{j}\left(x_{j}^{i}+w_{j}^{i}-x_{n_{i}+1}^{i}\right)+\sum_{j=1}^{n_{i}} \eta_{j}\left(y_{j}^{i}+h_{j}^{i}-y_{n_{i}+1}^{i}\right) \\
+ & \sum_{j=1}^{n_{i}} \sum_{e(p, q) \in G_{h j}} \lambda_{j p q}\left(x_{p}+w_{p}-x_{q}\right) \\
+ & \sum_{j=1}^{n_{i}} \sum_{e(p, q) \in G_{v j}} \mu_{j p q}\left(y_{p}+\frac{A_{p}}{w_{p}}-y_{q}\right) \\
+ & \sum_{j=1}^{n_{i}} r_{j}\left(x_{t_{j}}-w_{j}^{i}\right)+s_{j}\left(y_{t_{j}}-h_{j}^{i}\right)
\end{aligned}
$$

subject to $\quad L_{i} \leq w_{i} \leq U_{i}, \forall 1 \leq i \leq n$.

Let $Q(\overrightarrow{\mathcal{P}})$ denote the optimal value of $L R S /(\overrightarrow{\mathcal{P}})$. The Lagrangian dual problem $L D P$ of $C S$ can be defined as follows:

$$
\begin{array}{cc}
\text { Maximize } & Q(\overrightarrow{\mathcal{P}}) \\
\text { subject to } & \overrightarrow{\mathcal{P}} \geq 0
\end{array}
$$

Since $C S$ can be transformed into a convex problem, we can apply Theorem 6.2.4 of [3]. This implies that if $\overrightarrow{\mathcal{P}}$ is an optimal solution to $L D P$, the optimal solution of $L R S /(\overrightarrow{\mathcal{P}})$ will also optimize $C S$.

Consider the Lagrangian $\zeta$ of $C S$ defined as follows:

$$
\begin{aligned}
\zeta & =\alpha x_{n_{i}+1}^{i} y_{n_{i}+1}^{i}+\beta \sum_{e_{j}^{i} \in E^{i}} \varpi_{j}^{i}+\sum_{j=1}^{n_{i}} \kappa_{j}\left(x_{j}^{i}+w_{j}^{i}-x_{n_{i}+1}^{i}\right) \\
& +\sum_{j=1}^{n_{i}} \eta_{j}\left(y_{j}^{i}+h_{j}^{i}-y_{n_{i}+1}^{i}\right)+\sum_{j=1}^{n_{i}} \sum_{e(p, q) \in G_{h j}} \lambda_{j p q}\left(x_{p}+w_{p}-x_{q}\right) \\
& +\sum_{j=1}^{n_{i}} \sum_{e(p, q) \in G_{v j}} \mu_{j p q}\left(y_{p}+\frac{A_{p}}{w_{p}}-y_{q}\right)+\sum_{j=1}^{n_{i}} r_{j}\left(x_{t_{j}}-w_{j}^{i}\right) \\
& +s_{j}\left(y_{t_{j}}-h_{j}^{i}\right)+\sum_{i=1}^{n} u_{i}\left(L_{i}-w_{i}\right)+\sum_{i=1}^{n} v_{i}\left(w_{i}-U_{i}\right) .
\end{aligned}
$$

The Kuhn-Tucker conditions imply that the optimal solution of $C S$ must be at $\partial \zeta / \partial x_{p}=0, \partial \zeta / \partial y_{p}=0, \partial \zeta / \partial x_{n_{i}+1}^{i}=0$, and $\partial \zeta / \partial y_{n_{i}+1}^{i}=0$. Thus, we only need to consider the multipliers $\overrightarrow{\mathcal{P}}$ which satisfy these conditions. Therefore, for $1<p<n$,

$$
\begin{aligned}
\partial \zeta / \partial x_{p} & =\sum_{j=1}^{n_{i}}\left(\sum_{e(p, q) \in G_{h j}} \lambda_{j p q}-\sum_{e(q, p) \in G_{h j}} \lambda_{j q p}\right)=0, \\
\partial \zeta / \partial y_{p} & =\sum_{j=1}^{n_{i}}\left(\sum_{e(p, q) \in G_{h j}} \eta_{j p q}-\sum_{e(q, p) \in G_{h j}} \eta_{j q p}\right)=0,
\end{aligned}
$$

and

$$
\begin{aligned}
\partial \zeta / \partial x_{n_{i}+1}^{i} & =\alpha y_{n_{i}+1}^{i}-\sum_{j=1}^{n_{i}} \kappa_{j}=0, \\
\partial \zeta / \partial y_{n_{i}+1}^{i} & =\alpha x_{n_{i}+1}^{i}-\sum_{j=1}^{n_{i}} \eta_{j}=0 .
\end{aligned}
$$

Thus, we have $y_{n_{i}+1}^{i}=\frac{1}{\alpha} \sum_{j=1}^{n_{i}} \kappa_{j}$, and $x_{n_{i}+1}^{i}=\frac{1}{\alpha} \sum_{j=1}^{n_{i}} \eta_{j}$.

\subsection{Solving $L R S /(\overrightarrow{\mathcal{P}})$ and $L D P$}

Let $\Omega$ denote the set of multipliers $\overrightarrow{\mathcal{P}}$ satisfying Equations (6) and (7). We now consider solving the Lagrangian relaxation subproblem $L R S /(\overrightarrow{\mathcal{P}})$ for a given $\overrightarrow{\mathcal{P}} \in \Omega$, i.e. computing the dimension and coordinate of each module. First, we partially differentiate $\zeta$ with respect to $w_{i}$ to get an optimal value of $w_{i}$ such that $\zeta$ is minimized.

$$
\begin{aligned}
\partial \zeta / \partial w_{i}=\left(v_{p}-u_{p}\right) & +\sum_{j=1}^{n_{i}}\left(\sum_{q \in \text { out }_{G_{h j}}\left(v_{p}\right)} \lambda_{j} p q\right) \\
& -\sum_{j=1}^{n_{i}}\left(\sum_{q \in \text { out }_{G_{v j}}\left(v_{p}\right)} \mu_{j p q} \frac{A_{p}}{w_{p}^{2}}\right)=0 .
\end{aligned}
$$

Thus, we have

$$
w_{p}=\sqrt{\frac{\sum_{j=1}^{n_{i}} \sum_{q \in o u t_{G_{v j}}\left(v_{p}\right)} \mu_{j p q} A_{p}}{\left(v_{p}-u_{p}\right)+\sum_{j=1}^{n_{i}} \sum_{q \in o u t_{G_{h j}}\left(v_{p}\right)} \lambda_{j} p q}}
$$

where $\operatorname{out}_{G}(v)=\{u \mid e(v, u) \in E(G)\}$. Recall that $L_{p} \leq w_{p} \leq U_{p}, 1 \leq p \leq$ $n$. Thus, the optimal $w_{p}^{*}$ can be computed by $w_{p}^{*}=\min \left\{U_{p}, \max \left\{L_{p}, w_{p}\right\}\right\}$.

Since the dimension of each primitive module $\left(w_{p}\right.$ and $\left.h_{p}\right)$ has been determined, the dimension of each cluster module $\left(w_{j}^{i}\right.$ and $\left.h_{j}^{i}\right)$ can be computed by applying a longest path algorithm in $G_{h j}$ and $G_{v j}$. Then, we consider partial differentiation of $\zeta$ with respect to $x_{j}^{i}$ and $y_{j}^{i}$, giving the optimality conditions of $C S$. Therefore, for $1 \leq j \leq n_{i}$,

$\partial \zeta / \partial x_{j}^{i}=\beta\left(\sum_{\substack{i \\ e_{k}^{i} \supset\left\{m_{j}^{i}\right\}}} 2\left(\left|e_{k}^{i}\right|-1\right) x_{j}^{i}-\sum_{e_{k}^{i} \supset\left\{m_{j}^{i}\right\}} \sum_{m_{l}^{i} \in e_{k}^{i} \backslash\left\{m_{j}^{i}\right\}} x_{l}^{i}\right.$ 


$$
\begin{aligned}
+ & \left.\sum_{e_{k}^{i} \supset\left\{m_{j}^{i}\right\}} \sum_{m_{l}^{i} \in e_{k}^{i} \backslash\left\{m_{j}^{i}\right\}}\left(w_{j}^{i}-w_{l}^{i}\right)\right)=0 \\
\partial \zeta / \partial y_{j}^{i} & =\beta\left(\sum_{e_{k}^{i} \supset\left\{m_{j}^{i}\right\}} 2\left(\left|e_{k}^{i}\right|-1\right) y_{j}^{i}-\sum_{e_{k}^{i} \supset\left\{m_{j}^{i}\right\}} \sum_{m_{l}^{i} \in e_{k}^{i} \backslash\left\{m_{j}^{i}\right\}} y_{l}^{i}\right. \\
& \left.+\sum_{e_{k}^{i} \supset\left\{m_{j}^{i}\right\}} \sum_{m_{l}^{i} \in e_{k}^{i} \backslash\left\{m_{j}^{i}\right\}}\left(h_{j}^{i}-h_{l}^{i}\right)\right)=0
\end{aligned}
$$

where $\left|e_{k}^{i}\right|$ denotes the number of the pins of $e_{k}^{i}$

In Equation (8), there are $n_{i}$ equations with $n_{i}$ variables. Thus, we can apply the Gaussian elimination to solve these $n_{i}$ equations with $n_{i}$ variables to get the optimal value of $x_{j}^{i}$. In these $n_{i}$ equations, all coefficients of variables depend only on the net information (i.e., $e_{k}^{i}$ ). Since the net information is the same through the entire process, each variable can be solved by the same process. Hence, we can record the process of solving each variable during the first iteration (which takes cubic time), and then each subsequent computation will take only quadratic time by applying the same process. Similarly, we can compute the optimal value of $y_{j}^{j}$.

Next, we use a subgradient optimization method to search for the optimal $\overrightarrow{\mathcal{P}}$. Let $\overrightarrow{\mathcal{P}}$ be a multiplier at step $k$. We move $\overrightarrow{\mathcal{P}}$ to a new multiplier $\overrightarrow{\mathcal{P}}^{\prime}$ based on the subgradient direction:

$$
\begin{aligned}
\kappa_{j}^{\prime} & =\left[\kappa_{j}+\rho_{k}\left(x_{j}^{i}+w_{j}^{i}-x_{n_{i}+1}^{i}\right)\right]^{+} \\
\eta_{j}^{\prime} & =\left[\eta_{j}+\rho_{k}\left(y_{j}^{i}+h_{j}^{i}-y_{n_{i}+1}^{i}\right)\right]^{+} \\
\lambda_{j p q}^{\prime} & =\left[\lambda_{j p q}+\rho_{k}\left(x_{p}+w_{p}-x_{q}\right)\right]^{+} \\
\mu_{j p q}^{\prime} & =\left[\mu_{j p q}+\rho_{k}\left(y_{p}+\frac{A_{p}}{w_{p}}-y_{q}\right)\right]^{+},
\end{aligned}
$$

where $[x]^{+}=\max \{x, 0\}$ and $\rho_{k}$ is a step size such that $\lim _{k \rightarrow \infty} \rho_{k}=0$ and $\sum_{k=1}^{\infty} \rho_{k}=\infty$.

After updating $\overrightarrow{\mathcal{P}}$, we need to project $\overrightarrow{\mathcal{P}^{\prime}}$ to $\overrightarrow{\mathcal{P}_{*}} \in \Omega$, and then solve the Lagrangian relaxation subproblem $L R S /(\overrightarrow{\mathcal{P} *})$ by the above algorithm until the solution converges.

\subsection{Projecting Lagrange Multipiers}

We present a network flow based algorithm to check whether $\overrightarrow{\mathcal{P}}$ belongs to $\Omega$ and to project $\overrightarrow{\mathcal{P}}$ to $\overrightarrow{\mathcal{P}} * \in \Omega$, if $\overrightarrow{\mathcal{P}} \notin \Omega$. Further, an increamental update technique is employed to make the maximum flow computation more efficient. For each cluster module $m_{c}$, we first create two networks $N_{h c}$ (for $G_{h c}$ ) and $N_{v c}$ (for $G_{v c}$ ) as follows:

- For each $v_{i} \in V\left(G_{h c}\right)\left(V\left(G_{v c}\right)\right)$, create a vertex $v_{i}^{\prime}$ in $N_{h c}\left(N_{v c}\right)$, and make $v_{s}^{\prime}$ and $v_{t}^{\prime}$ as the source and sink, respectively.

- For each $e(p, q) \in E\left(G_{h c}\right)\left(E\left(G_{v c}\right)\right)$, create a corresponding edge $e\left(p^{\prime}, q^{\prime}\right)$ with capacity $\lambda_{c p q}\left(\mu_{c p q}\right)$ in $N_{h c}\left(N_{v c}\right)$.

We apply the maximum flow computation on the networks to check whether $\overrightarrow{\mathcal{P}}$ belongs to $\Omega$. The maximum flow computation finds an augmenting path from $\gamma_{s}$ to $v_{t}^{\prime}$ and then pushes flow on it until no argument path can be found. Let $\operatorname{cap}(v, u)$ and $f \operatorname{low}(v, u)$ denote the capacity and flow on the edge $e(v, u)$. An edge $e(v, u)$ is saturated if its capacity equals the flow (i.e., $\operatorname{cap}(v, u)=\operatorname{flow}(v, u)$ ).

Theorem 2 If all edges in the networks are saturated, $\overrightarrow{\mathcal{P}} \in \Omega$.

If $\overrightarrow{\mathcal{P}}$ does not belong to $\Omega$, we project $\overrightarrow{\mathcal{P}}$ to $\overrightarrow{\mathcal{P}_{*}}$ by restoring the flow flow $\left(p^{\prime}, q^{\prime}\right)$ of each edge $e\left(p^{\prime}, q^{\prime}\right)$ in $N_{h c}\left(N_{v c}\right)$ to $\lambda_{c p q}\left(\mu_{c p q}\right)$ for each $m_{c}$.

\section{Theorem $3 \overrightarrow{\mathcal{P} *} \in \Omega$}

The projection process greatly affects the efficiency of the entire optimization, since there may be $O\left(n^{2}\right)$ edges in the worst case. Thus, we employ an incremental flow update technique to speed up the max-flow computation after updating $\overrightarrow{\mathcal{P}}$ and its corresponding capacity. Figure 6 gives an algorithm for the incremental network update. Lines 1-2 check whether each edge $e\left(p^{\prime}, q^{\prime}\right)$ violates the capacity constraint (i.e., $0 \leq f l o w\left(p^{\prime}, q^{\prime}\right) \leq \operatorname{cap}\left(p^{\prime}, q^{\prime}\right)$ ). Lines 3-9 fix the overflow on $e\left(p^{\prime}, q^{\prime}\right)$, if an edge $e\left(p^{\prime}, q^{\prime}\right)$ violates its capacity constraint. Finally, Line 10 computes a maximum flow again

Note that, for efficiency consideration, we may perform Lagrangian relaxation only at the higher levels of the multilevel framework (when the number of modules become small enough for Lagrangian relaxation). To do so, however, we still need to pass the information of the aspect ratio for each soft module level by level.

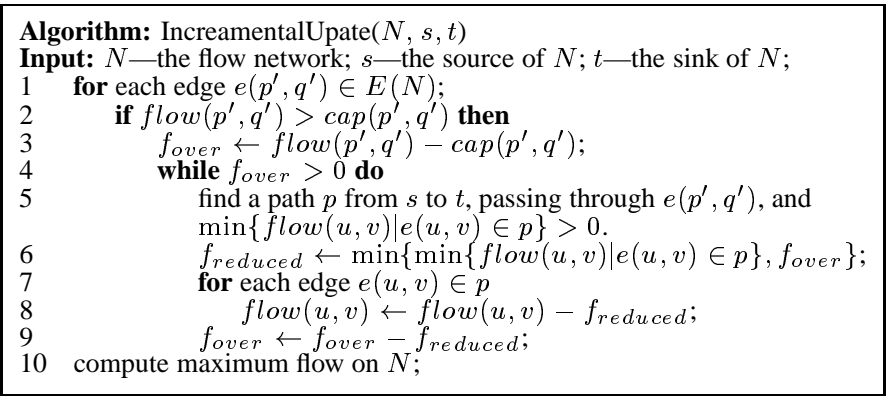

Figure 6: The increamental update algorithm.

\section{Experimental Results}

We implemented the MB*-tree algorithm for hard modules in the C++ language on a $450 \mathrm{MHz}$ SUN Ultra 60 workstation with $2 \mathrm{~GB}$ memory. The package is available at http://cc.ee.ntu.edu.tw/ ywchang/research.html

Columns $1,2,3$, and 4 of Table 1 lists the names of the benchmark circuits, the number of modules, the number of nets, and the total area of modules in the circuits, respectively. ami49 is the largest MCNC benchmark circuit used in the previous works $[5,9]$ for comparative study. To test the capability of existing methods, we created ten synthetic circuits, named ex ami49 $x$, by duplicating the modules and nets of ami49 by $x$ times. The largest circuit ex_ami49_200 contains 9,800 modules and 81,600 nets

Table 1 also shows the results for ex_ami49_x by optimizing area alone $(\gamma=$ 1.0 and $\delta=0.0$ ). Columns 5,6, and 7 give the resulting area, the dead space, and the runtime for our $\mathrm{MB}^{*}$-tree, respectively. The remaining columns list the results for the famous previous works, sequence pair [18], O-tree [9], and $\mathrm{B}^{*}$-tree [5]. Note that the $\mathrm{B} *$-tree package we used here is the September 2000 version, $B^{*}$ tree-v1.0, available also at http://cc.ee.ntu.edu.tw/ ywchang/research.html. It runs $50 \mathrm{X}-100 \mathrm{X}$ faster and achieves better area utilization than the $\mathrm{B}^{*}$-tree package reported in [5]. As shown in the table, our MB*-tree algorithm obtained a dead space of only $2.78 \%$ for ami49 in only 0.4 min runtime while B*-tree-v1.0 reported a dead space of $3.53 \%$ using 0.25 min runtime. Further, the experimental results for larger circuits show that the $\mathrm{MB}^{*}$-tree scales very well as the circuit size increases while the previous works, sequence pair, $\mathrm{O}$-tree, and $\mathrm{B} *$-tree, do not. For circuit sizes ranging from 49 to 9,800 modules and from 408 to 81,600 nets, the MB*-tree consistently obtains high-quality floorplans with dead spaces of less than $3.72 \%$ in empirically linear runtime, while sequence pair, O-tree, and $\mathrm{B}^{*}$-tree can handle only up to 196,98 , and 1,960 modules in the same amount of time and result in dead spaces of as large as 13.00\% (@196 modules), 12.29\% (@ 98 modules), and 27.33\% (@1960 modules), respectively. As shown in Table 1, the resulting dead spaces for the MB*-tree is almost independent of the circuit sizes, which proves the high scalability of the $\mathrm{MB}^{*}$-tree. In contrast, the dead spaces for the non-hierarchical previous works all grow dramatically as the circuit size increases. In particular, the empirical runtime of the $\mathrm{MB}^{*}$-tree approaches linear in the circuit size while the other previous works cannot handle large-scale designs. Figure 7 shows the layout for the largest circuit ex ami49-200 obtained by MB*-tree in 256 min CPU time. It has a dead space of only $3.44 \%$. Note that this circuit is not feasible to the previous works $[5,9,18]$.

Table 2 shows the comparisons for area optimization alone $(\gamma=1.0, \delta=$ $0.0)$, wirelength optimization alone $(\gamma=0.0, \delta=1.0)$, and simultaneous area and wirelength optimization $(\gamma=0.5, \delta=0.5)$ among sequence pair (SP), $\mathrm{B}^{*}$-tree, and $\mathrm{MB}^{*}$-tree based on the circuit industry (whose total area $=658.04$ $\mathrm{mm}^{2}$ ). The circuit industry is a $0.18 \mu \mathrm{m}, 1 \mathrm{GHz}$ industrial design with 189 modules, 20 million gates, and 9,777 center-to-center interconnections. It is a large chip design and consists of three "tough" modules with aspect ratios greater than 19 (and as large as 36). (Note that we do not have the results for O-tree for this experiment because the data industry cannot be fed into the O-tree package.) In each entry of the table, we list the best/average values obtained in ten runs of simulated annealing, using a random seed for each run. For the column "Time," we report the runtime for obtaining the best value and the average runtime of the ten runs. As shown in the table, our MB*-tree algorithm obtained significantly better silicon area and wirelength than sequence pair and $\mathrm{B}^{*}$-tree in all tests. For area optimization, $\mathrm{MB}^{*}$-tree can obtain a dead space of only $2.11 \%$ while sequence pair (B*-tree) results in a dead space of at least $28.1 \%(12.9 \%)$. For wirelength optimization, $\mathrm{MB}^{*}$-tree can obtain a total wirelength of only $56631 \mathrm{~mm}$ while sequence pair (B*-tree) requires a total wirelength of at least $81344 \mathrm{~mm}(113216$ $m m$ ). For simultaneous area and wirelength optimization, $\mathrm{MB}^{*}$-tree also obtains the best area and wirelength. The results show the effectiveness of our MB*-tree algorithm. For the runtimes, $\mathrm{MB}^{*}$-tree is larger than $\mathrm{B}^{*}$-tree and $\mathrm{SP}$ for wirelength optimization. (For area optimization, MB*-tree runs faster than SP.) This is reasonable because it took much longer to obtain significantly better results and the multulevel process incurred some overhead. Nevertheless, as shown in Table 1, both SP and $\mathrm{B}^{*}$-tree do not scale well to the instances with a large number of modules (and thus their runtimes increase dramatically when the number of modules grows into hundreds). The resulting layout of industry for simultaneous area and wirelength optimization using MB*-tree is shown in Figure 8. 


\begin{tabular}{|c|c|c|c|c|c|c|c|c|c|c|c|c|c|c|c|}
\hline \multirow[b]{2}{*}{ Circuit } & \multirow[b]{2}{*}{$\begin{array}{c}\# \\
\text { modules }\end{array}$} & \multirow[b]{2}{*}{$\begin{array}{c}\# \\
\text { nets }\end{array}$} & \multirow[b]{2}{*}{$\begin{array}{c}\text { Total } \\
\text { area } \\
\left(\mathrm{mm}^{2}\right)\end{array}$} & \multicolumn{3}{|c|}{ MB*-tree } & \multicolumn{3}{|c|}{ Sequence Pair [18] } & \multicolumn{3}{|c|}{ O-tree [9] } & \multicolumn{3}{|c|}{$\mathrm{B} *$-tree } \\
\hline & & & & $\begin{array}{c}\text { Area } \\
\left(\mathrm{mm}^{2}\right)\end{array}$ & $\begin{array}{l}\text { Dead } \\
\text { space } \\
(\%)\end{array}$ & $\begin{array}{l}\text { Time } \\
(\mathrm{min})\end{array}$ & $\begin{array}{c}\text { Area } \\
\left(m m^{2}\right)\end{array}$ & $\begin{array}{c}\text { Dead } \\
\text { space } \\
(\%)\end{array}$ & (min) & $\begin{array}{c}\text { Area } \\
\left(\mathrm{mm}^{2}\right)\end{array}$ & $\begin{array}{c}\text { Dead } \\
\text { space } \\
(\%)\end{array}$ & $\begin{array}{l}\text { Time } \\
\text { (min) }\end{array}$ & $\begin{array}{c}\text { Area } \\
\left(\mathrm{mm}^{2}\right)\end{array}$ & $\begin{array}{c}\text { Dead } \\
\text { space } \\
(\%)\end{array}$ & $\begin{array}{l}\text { Time } \\
(\mathrm{min})\end{array}$ \\
\hline ami49 & 49 & 408 & 35.445 & 36.46 & 2.78 & 0.4 & 38.89 & 8.87 & 6.86 & 36.77 & 3.61 & 10.46 & 36.74 & 3.53 & 0.25 \\
\hline ex_ami49_2 & 98 & 816 & 70.890 & 72.72 & 2.51 & 2.5 & 80.27 & 11.69 & 45.51 & 80.82 & 12.29 & 70.56 & 73.11 & 3.03 & 1.26 \\
\hline ex_ami49_4 & 196 & 1632 & 141.780 & 145.73 & 2.70 & 2.6 & 162.97 & 13.00 & 309.00 & 155.76 & 9.86 & 179.23 & 151.31 & 6.60 & 4.73 \\
\hline ex_ami49_10 & 490 & 4080 & 354.454 & 364.14 & 2.66 & 5.4 & NR & NR & NR & NR & NR & $\mathrm{NR}$ & 407.32 & 12.98 & 19.25 \\
\hline ex_ami49_20 & 980 & 9160 & 708.908 & 730.95 & 3.02 & 15.6 & NR & NR & NR & NR & $\mathrm{NR}$ & NR & 870.45 & 18.55 & 23.48 \\
\hline ex_ami49_40 & 1960 & 16320 & 1417.816 & 1472.62 & 3.72 & 24.8 & NR & NR & NR & NR & NR & NR & 1951.04 & 27.33 & 53.21 \\
\hline ex_ami49_60 & 2940 & 24480 & 2126.724 & 2205.86 & 3.58 & 42.2 & NR & NR & NR & NR & NR & NR & NR & NR & NR \\
\hline ex_ami49_80 & 3920 & 32640 & 2835.632 & 2943.72 & 3.67 & 57.0 & NR & NR & NR & NR & NR & NR & NR & NR & NR \\
\hline ex_ami49_100 & 4900 & 40800 & 3544.540 & 3671.42 & 3.45 & 51.6 & NR & NR & NR & NR & NR & NR & NR & NR & NR \\
\hline ex_ami49_150 & 7350 & 61200 & 5316.750 & 5505.34 & 3.42 & 142.2 & NR & NR & NR & NR & NR & NR & NR & NR & NR \\
\hline ex_ami49_200 & 9800 & 81600 & 7089.080 & 7341.91 & 3.44 & 256.2 & NR & NR & NR & NR & NR & NR & NR & NR & NR \\
\hline
\end{tabular}

Table 1: Comparisons for area, dead space, and runtime among MB*-tree, Sequence pair, O-tree, and B*-tree. NR: No result obtained within 5-hr CPU time on SUN Sparc Ultra 60.

\begin{tabular}{|c|c|c|c|c|c|c|c|c|c|}
\hline \multirow[t]{2}{*}{ Package } & \multicolumn{3}{|c|}{$\begin{array}{c}\text { Area optimization } \\
(\gamma=1.0, \delta=0.0)\end{array}$} & \multicolumn{2}{|c|}{$\begin{array}{l}\text { Wirelength optimization } \\
(\gamma=0.0, \delta=1.0)\end{array}$} & \multicolumn{4}{|c|}{$\begin{array}{l}\text { Simultaneous area and wirelength optimization } \\
(\gamma=0.5, \delta=0.5)\end{array}$} \\
\hline & $\begin{array}{c}\text { Area } \\
\left(\mathrm{mm}^{2}\right) \\
\end{array}$ & $\begin{array}{c}\text { Dead space } \\
(\%)\end{array}$ & $\begin{array}{l}\text { Time } \\
(\min )\end{array}$ & $\begin{array}{c}\text { Wirelength } \\
(\mathrm{mm})\end{array}$ & $\begin{array}{l}\text { Time } \\
(\mathrm{min}) \\
\end{array}$ & $\begin{array}{c}\text { Area } \\
\left(m m^{2}\right) \\
\end{array}$ & $\begin{array}{c}\text { Dead space } \\
(\%)\end{array}$ & $\begin{array}{c}\text { Wirelength } \\
(\mathrm{mm})\end{array}$ & $\begin{array}{l}\text { Time } \\
(\mathrm{min}) \\
\end{array}$ \\
\hline $\mathrm{SP}$ & $914.5 / 988.0$ & $28.1 / 33.2$ & $46.8 / 35.31$ & $713216 / 122609$ & $31.3 / 37.3$ & $7104.2 / 1182.5$ & $=40.4 / 44.3$ & $736001 / 159340$ & $46.4 / 37.3$ \\
\hline $\mathrm{B}^{*}$-tree & 755.71876 .6 & $12.9 / 24.7$ & $0.2 / 0.1$ & $81344 / 91169$ & $12.6 / 9.1$ & 834.71982 .8 & $21.2 / 32.5$ & $104558 / 112200$ & $14.4 / 9.11$ \\
\hline $\mathrm{MB}^{*}$-tree & $671.9 / 740.8$ & $2.1 / 3.1$ & $71.5 / 6.6$ & $56631 / 61698$ & $346.1 / 204.7$ & $716.3 / 740.8$ & $8.1 / 11.1$ & 67786667541 & $221.3 / 220.2$ \\
\hline SP:MB*-tree & $1.36 / 1.34$ & $13.38 / 10.71$ & 4.0715 .35 & $2.00 / 1.98$ & $0.09 / 0.18$ & $1.54 / 1.60$ & $4.99 / 3.99$ & $2.01 / 2.36$ & $0.21 / 0.17$ \\
\hline $\mathrm{B}^{*}$-tree $: \mathrm{MB}^{*}$-tree & $1.13 / 1.18$ & $6.14 / 7.97$ & $0.02 / 0.02$ & $1.44 / 1.48$ & $0.04 / 0.05$ & $1.17 / 1.33$ & $2.62 / 2.93$ & $1.54 / 1.66$ & $0.07 / 0.04$ \\
\hline
\end{tabular}

Table 2: Comparisons for area optimization alone, wirelength optimization alone, and simultaneous area and wirelength optimization among sequence pair (SP), $\mathrm{B}^{*}$-tree, and $\mathrm{MB}^{*}$-tree based on the circuit industry. In each entry, both the best/average values obtained in ten runs of simulated annealing are reported. The last two rows give the ratios of the results ( $\mathrm{SP}$ to $\mathrm{MB}^{*}$-tree and $\mathrm{B}^{*}$-tree to $\mathrm{MB}^{*}$-tree).

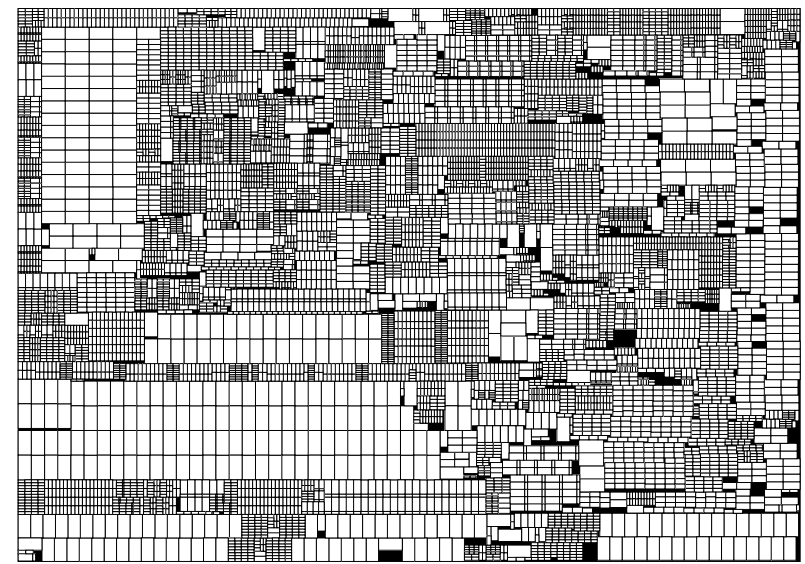

Figure 7: The layout of ex_ami49_200 (9,800 modules, 81,600 nets). Dead space $=3.44 \%$.

\section{Concluding Remarks}

We have presented the $\mathrm{MB}^{*}$-tree based multilevel framework to handle the floorplanning and packing for large-scale modules. Experimental results have shown that the $\mathrm{MB}^{*}$-tree scales very well as the circuit size increases. The capability of the $\mathrm{MB}^{*}$-tree shows its promise in handling large-scale designs with complex constraints. We propose to explore the floorplanning/placement problem with large-scale rectilinear and mixed sized modules/cells as well as buffer-block planning for interconnect-driven floorplanning in the future.

\section{References}

[1] C. J. Alpert, J. H. Hu, S. S. Sapatnekar, and P. G. Villarrubia, "A practical methodology for early buffer and wire resource allocation," Proc. of DAC, pp. 189-194, 2001.

[2] C. J. Alpert, J.-H. Huang, and A. B. Kahng, "Multilevel circuit partitioning," IEEE Trans. CAD, vol. 17, no. 8, pp. 655-667, August 1998.

[3] M. S. Bazaraa, H. D. Sherali, C. M. Shetty, Nonlinear Programming Theory and Algorithms, 1993.

[4] T. F. Chan, J. Cong, T. Kong, J. R. Shinnerl, "Multilevel optimization for large-scale circuit placement," Proc. of ICCAD, pp. 171-176, 2000.

[5] Y.-C. Chang, Y.-W. Chang, G.-M. Wu and S.-W. Wu, "B*-Trees: A new representation for non-slicing floorplans," Proc. of DAC, pp. 458-463, 2000.

[6] J. Cong, J. Fang, and Y. Zhang, "Multilevel approach to full-chip gridless routing," Proc. of ICCAD, pp. 396-403, 2001.

[7] J. Cong, T. Kong and D. Z. Pan, "Buffer Block Planning for Interconnect-Driven Floorplanning," Proc. of ICCAD, pp. 358-363, 1999.

[8] J. Cong, M. Xie, and Y. Zhang, "An enhanced multilevel routing system," Proc. of ICCAD, pp. 51-58, 2002.

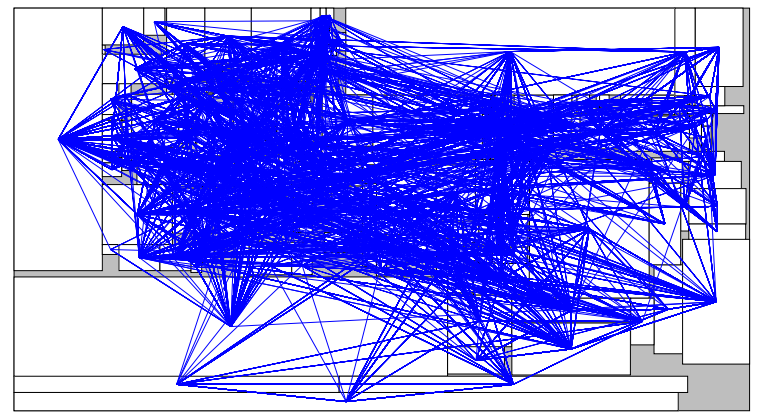

Figure 8: The layout of industry by simultaneously optimizing area and wirelength $(\gamma=0.5, \delta=0.5)$. CPU time $=5234 \mathrm{sec}$, Area $=716263680 \mu \mathrm{m}^{2}$, Total wirelength $=67786296 \mu \mathrm{m}$, Dead space $=8.14 \%$.

[9] P.-N. Guo, C.-K. Cheng, and T. Yoshimura, "An O-tree representation of non-slicing floorplan and its applications," Proc. of DAC, pp. 268-273, 1999.

[10] B. Hendrickson and R. Leland, "A multilevel algorithm for partitioning graph," Proc. of Supercomputing, 1995.

[11] H.-R. Jiang, Y.-W. Chang, J.-Y. Jou, and K.-Y. Chao, "Simultaneous floorplanning and buffer block planning," Proc. of ASP-DAC, pp. 431-434, 2003.

[12] G. Karypis and V. Kumar, "Multilevel $k$-way hypergraph partitioning," Proc. of DAC, pp. 343-348, 1999

[13] S.-M. Li, Y.-H. Cherng, and Y.-W. Chang, "Noise-aware buffer planning for interconnect-driven floorplanning," Proc. of ASP-DAC, pp. 423-426, 2003.

[14] J.-M. Lin and Y.-W. Chang, "TCG: A transitive closuer graph based representation for non-slicing floorplans," Proc. of DAC, pp. 764-769, 2001.

[15] J.-M. Lin and Y.-W. Chang, "TCG-S: Orthogonal coupling of $\mathrm{P} *$-admissible representations for general floorplans," Proc. of DAC, pp. 842-847, 2002.

[16] J.-M. Lin, Y.-W. Chang, and S.-P. Lin, "Corner sequence: A P-admissible floorplan representation with a worst-case linear-time packing scheme," IEEE Trans. VLSI Systems, 2003.

[17] S.-P. Lin and Y.-W. Chang, "A novel framework for multilevel routing considering routability and performance," Proc. of ICCAD, pp. 44-50, 2002.

[18] H. Murata, K. Fujiyoshi, S. Nakatake, and Y. Kajitani, "Rectangle-packing based module placement," Proc. of ICCAD, pp. 472-479, 1995.

[19] S. Nakatake, K. Fujiyoshi, H. Murata, and Y. Kajitani, "Module placement on BSGstructure and IC layout applications," Proc. of ICCAD, pp. 484-491, 1996

[20] R. H. J. M. Otten, “Automatic floorplan design,” Proc. of DAC, pp. 261-267, 1982.

[21] P. Sarkar, V. Sundararaman and C.K. Koh, "Routability-driven repeater block planning for interconnect-centric floorplanning," Proc. of ISPD, 2000

[22] D. F. Wong and C. L. Liu, "A new algorithm for floorplan design," Proc. of DAC, pp. 101-107, 1986

[23] F.Y. Young, C. C.N. Chu, W.S. Luk, and Y.C. Wong, "Floorplan area minimization using Lagrangian relaxation," Proc. of ISPD., pp. 174-179, 2000. 